\title{
13 Adverbial eingeleitete Verbletztsätze
}

1 Beispiele

2 Terminologie

3 Markierungsebenen

4 Theoretische Probleme

5 Zusammenfassung

6 Literatur

\section{Beispiele}

Die durch Fettsetzung ausgezeichneten Sätze in den folgenden Beispielen sind typische Exemplare des hier zu behandelnden Satztyps:

(1) Wenn der Herbst mit seinen langen Abenden kommt, wird es Zeit für alle Leseratten, sich mit Lesestoff einzudecken. (Niederösterreichische Nachrichten, 15. 09. 2008)

(2) Die Lager sind auf jeden Fall gefüllt, weil der Sommer noch nicht das Gelbe vom Ei war. (Frankfurter Allgemeine, 26. 07. 2005)

(3) Der Mann hat, wenn man sich vor Augen führt, welches Erbe er antrat, tatsächlich einen guten Job gemacht. (Mannheimer Morgen, 20. 01. 2010)

Die Sätze sind (A) durch eine nicht flektierbare, unveränderliche Einheit wie wenn oder weil eingeleitet, die (B) selbst kein Satzglied des von ihr eingeleiteten Satzes ist, und (C) das Finitum befindet sind am Satzende (daher Verbletzt). Die Beispiele zeigen auch, dass Sätze dieses Satztyps variabel sind in Bezug auf ihre Stellung im Gesamtsatz (Merkmal (D): Sie können dem Hauptsatz vorangehen und dabei das Vorfeld im Gesamtsatz einnehmen (vgl. (1)), sie können aber auch dem Hauptsatz nachfolgen und dann das Nachfeld des Gesamtsatzes besetzen (vgl. (2)). Seltener werden sie auch in das Mittelfeld des Hauptsatzes eingeschoben (vgl. (3)). Beispiel (3) zeigt exemplarisch auch, dass Sätze dieses Typs selbst Matrixsatz für weitere Sätze sein können, im Beispiel für den durch welches Erbe eingeleiteten Satz.

Mit den Beispielen ist auch angedeutet, dass die „Verbletztsatz-Einleiter“ in der Regel eine bestimmte semantische Beziehung zwischen dem Inhalt des Verbletztsatzes und des Hauptsatzes (oder allgemeiner des Matrixsatzes) ausdrücken: wenn drückt eine temporale oder konditionale Beziehung aus, weil eine kausale. Weitere Verhältnisse, die typischerweise durch diesen Satztyp ausgedruickt wer- 
den sind: Finalität (damit), Konzessivität (obgleich), Konsekutivität (sodass) oder auch Adversativität (während) usw. Es herrscht in der Forschung und Grammatikschreibung keine Einigkeit darüber, wie viele solche Verhältnisse es gibt und wie sie zu unterscheiden sind. Pittner (Adverbialsätze in diesem Band) orientiert sich an der klassischen Einteilung, fasst jedoch ähnlich wie die IDS-Grammatik Kausalität, Konsekutivität, Konzessivität und Finalität sowie auch das Irrelevanzkonditionale zur Großklasse der Grund-Folge-Relationen zusammen. Klar ist in jedem Fall, dass die Einleitungselemente verantwortlich sind für die Art des semantischen Verhältnisses zwischen den Inhalten der Teilsätze. Sie haben somit „lexikalische Bedeutung“(vgl. Eisenberg 2006: 204).

Für unseren Zusammenhang ist die Abgrenzung gegenüber anderen Verbletztsatztypen wesentlich, zum einen gegenüber Sätzen, die mit einem $w$ - oder $d$-Element eingeleitet werden, zum anderen gegenüber Sätzen mit dass oder $o b$ als Einleitungselement. In beiden Fällen fehlt ein Einleitungselement mit einer lexikalischen Bedeutung. Allerdings gibt es einen Übergangsbereich zwischen w-Elementen, insbesondere $w$-Adverbien wie wo, wobei, worauf, warum und den Verbletztsatz-Einleitern, die hier interessieren, auf den wir weiter unten eingehen (vgl. Abschnitt 3.6).

\section{Terminologie}

Traditionell werden Verbletztsatz-Einleiter dieser Art als ,Konjunktionen' bezeichnet, die von ihnen eingeleiteten Sätze als ,Konjunktionalsätze‘. Um diese Art Konjunktionen etwa von und, oder, denn zu unterscheiden, spricht man genauer auch von ,unterordnenden' bzw. ,subordinierenden Konjunktionen' gegenüber ,nebenordnenden“ bzw. ,koordinierenden Konjunktionen‘. Da die beiden Konjunktionenarten und die von ihnen eingeleiteten Sätze sich syntaktisch (und semantisch oder pragmatisch) stark unterscheiden, werden neuerdings häufig Bezeichnungen ohne einen gemeinsamen Oberbegriff bevorzugt. Man unterscheidet dann Konjunktionen bzw. Konjunktoren (wie und, oder mit Verbzweitstellung) und Subjunktionen oder Subjunktoren (mit Verbletztstellung). Entsprechend werden die Satztypen als Subjunktionalsätze bzw. als Subjunktorsätze bezeichnet. In HDK-1 wird der Terminus ,Subjunktorphrase ${ }^{6}$ anstelle von Subjunktorsatz gewählt, weil der Subjunktor als syntaktisch determinierendes Element der Konstruktion, als ihr ,Kopf“ zu werten ist, nicht der von ihr determinierte Satz. Die folgende Tabelle gibt einen Überblick über die in Grammatiken und Handbüchern verwendeten Termini: 


\section{(subordinierende) Subjunktion Subjunktor unterordnende) \\ Konjunktion}

Konjunktionssatz

Konjunktionalsatz

Subjunktionalsatz

Subjunktorsatz
Paul (1920)

Eisenberg (2006)
Duden (2009),

Eroms (2000)
Engel (2004), Fabricius-Hansen (2007), IDS-Grammatik

HDK-1

Tab. 1: Überblick zur Terminologie

Mit der rein formbezogenen Bezeichnung, Subjunktorsatz ist (z.B. nach IDSGrammatik) keine Abgrenzung gegenüber den dass- und $o b$-Sätzen geleistet: Auch diese „subordinieren“ und fordern Verbletztstellung. Diese Abgrenzung kann offensichtlich nur über ein semantisches Kriterium erfolgen oder ein Kriterium nach den syntaktischen Funktionen der Sätze. Traditionell geschieht Letzteres. Man spricht mit Blick auf Sätze wie (1) bis (3) von,Adverbialsätzen` und meint damit die syntaktische Funktion der Adverbialbestimmung. Der weil-Satz ist ebenso wie etwa der Ausdruck aus diesem Grund bzw. deswegen, mit dem er austauschbar ist, in dem Gesamtsatz eine adverbiale Bestimmung. Als adverbiale Bestimmung ist der Subjunktorsatz auf den gesamten Restsatz bezogen (vgl. etwa zur Definition bei Eisenberg 2006: 49). Wie in Abschnitt 3.5 gezeigt werden wird, ist die Adverbialbestimmung zwar die typische syntaktische Funktion dieser Sätze, aber nicht die einzig mögliche. Eine differenzierende Bezeichnung mit Bezug auf die semantische Funktion der Sätze, unabhängig von der syntaktischen Funktion, erscheint daher angemessener. In der Duden-Grammatik (2009: § 1689) wird hier von ,Verhältnissatz' im Gegensatz zum durch dass/ob eingeleiteten ,Inhaltssatz' gesprochen. Unter diesen Begriff fallen aber auch konditionale und andere Verberstsätze wie in Beispiel (4), das Beispiel (1) weitgehend entspricht:

(4) Kommt der Herbst mit seinen langen Abenden, wird es Zeit für alle Leseratten, sich mit Lesestoff einzudecken.

Man müsste daher genauer von, Verhältnissätzen mit Subjunktor oder ,Subjunktor-Verhältnissätzen' sprechen. Wir schlagen eher den umschreibenden Terminus: ,durch einen Subjunktor mit lexikalischer Bedeutung eingeleitete Verbletztsätze vor, oder kürzer: ,Verbletztsätze mit semantischem Subjunktor". Damit berufen wir uns auf die Analogie zu ,semantischen' Präpositionen wie auf 
oder vor (z. B. in Das Buch liegt auf dem Tisch, Er kommt vor 12 Uhr) gegenüber, formalen' Präpositionen wie dt. von in das Buch von Hans oder frz. de in chemin de fer. In einer entsprechenden Beziehung stehen auch die ,semantischen" Subjunktoren weil, wenn usw. gegenüber den formalen dass und $o b .{ }^{1}$ Wo keine Missverständnisse aufkommen können, sprechen wir auch weiterhin kurz von Subjunktorsätzen. Wir sehen damit auch von der in generativen Ansätzen vorherrschenden Begrifflichkeit ab, nach der dass und $o b$ als ,Komplementierer (,complementizer') von den übrigen Verbletztsatz-Einleitern abgegrenzt werden.

\section{Markierungsebenen}

\subsection{Reihenfolgemerkmale}

Zu unterscheiden ist die interne Reihenfolge im Subjunktorsatz von der Stellung des Subjunktorsatzes im Gesamtsatz, also der externen Topologie.

Im Subjunktorsatz besetzt der Subjunktor entsprechend der Feldereinteilung des deutschen Satzes die linke Satzklammer, das Vorfeld entfällt, im Mittelfeld erscheinen in der Regel alle Satzglieder außer dem Prädikat, darunter auch das Subjekt. Die Reihenfolge der einzelnen Mittelfeldkonstituenten folgt den generell hierfür geltenden Prinzipien. In der rechten Satzklammer werden das finite Verb (i) und gegebenenfalls die anderen das Prädikat bildende infiniten Teile des Verbalkomplex positioniert (ii). Eine Nachfeldbesetzung ist nicht ausgeschlossen, aber markiert (iii).

\begin{tabular}{|c|c|c|c|}
\hline linke Satzklammer & Mittelfeld & rechte Satzklammer & Nachfeld \\
\hline (i) wenn & $\begin{array}{l}\text { [der Herbst }]_{S u b i} \\
{[\text { mit seinen langen Abenden }]_{A d v}}\end{array}$ & kommt & \\
\hline (ii) wenn & $\begin{array}{l}\text { [der Herbst }]_{S u b i} \\
\text { [mit seinen langen Abenden }]_{A d v}\end{array}$ & gekommen sein wird & \\
\hline (iii) wenn & {$[\text { der Herbst }]_{S u b i}$} & kommt & $\begin{array}{l}\text { [mit seinen langen } \\
\text { Abenden] }\end{array}$ \\
\hline
\end{tabular}

Tab. 2: Topologie der Verbletztsätze mit semantischem Subjunktor

1 Die Existenz von zwei formalen Subjunktoren zeigt aber, dass immerhin jeweils vergleichsweise abstrakte semantische Werte ausgedrückt werden. Diese beziehen sich auf den Wahrheitsstatus der durch den Nebensatz ausgedrückten Proposition; vgl. dazu IDS-Grammatik (S. 2253-2258) sowie Axel, Unselbstständiger dass- und ob-VL-Satz, in diesem Band, Abschnitt 1.4, und Pittner, Akkusativobjektsätze, in diesem Band, Abschnitt 2. 
Die Topologie insbesondere des Mittelfeldes hängt wie bei anderen Satztypen eng mit der Fokusstruktur, also der Vordergrund-Hintergrund-Gliederung zusammen, mittelbar auch mit der Intonation, die entscheidend an der Fokusstruktur beteiligt ist (vgl. auch Abschnitt 3.4) zur externen Topologie.

Bereits anhand der Beispiele (1) bis (3) wurde, gezeigt, dass Verbletztsätze mit semantischem Subjunktor im Allgemeinen im Vorfeld, Nachfeld oder (seltener) im Mittelfeld ihres Matrixsatzes erscheinen können. Alle drei Reihenfolgebeziehungen sind im Prinzip mit den drei später zu erläuternden fokalen Strukturen vereinbar (vgl. HDK-1: 371ff.):

Matrixsatz und Subjunktorsatz liefern beide Vordergrundinformation.

Der Matrixsatz liefert Hintergrund-, der Subjunktorsatz Vordergrundinformation.

Der Subjunktorsatz liefert Hintergrund-, der Matrixsatz Vordergrundinformation.

Wenn beide Sätze Vordergrundinformation liefern, also neue oder hervorzuhebende Information, scheint allerdings die Reihenfolge Subjunktorsatz im Vorfeld des Matrixsatzes zu überwiegen, während sonst die Nachfeldposition dominant ist.

Eine Reihe von Verbletztsatzeinleitern beschränken jedoch die Stellungsmöglichkeiten in der Weise, dass nur die Position im Nachfeld des Matrixsatzes zugelassen ist, wie in:

(5) Der August kann ja noch immer sehr heiß werden, so dass der Sommer dann ganz anders als bislang in Erinnerung bleibt. (Nürnberger Nachrichten, 26. 07. 2004)

(6) Der Winter geht von Mai bis November und ist recht trocken, wohingegen der Sommer von November bis Mai recht feucht ist. (Wikipedia-Eintrag zu Mauritius)

Hierher gehören unter anderem folgende Einleitungselemente: so dass/sodass, als dass, zumal sowie eine ganze Reihe von mit einem $w$-Element zusammengesetzte Formen wie: weshalb, weswegen, wobei, wodurch, wohingegen. In HDK-1 werden diese Elemente als ,Postponierer' bezeichnet und von den Subjunktoren getrennt (vgl. auch zur Liste der Postponierer HDK-1: 418). Die durch einen Postponierer eingeleiteten Sätze werden dort als ,Postponiererphrasen“ bezeichnet. Wir folgen diesem Vorschlag hier nicht: Auch sie betrachten wir als ,Verbletztsätze mit semantischem Subjunktor‘. Allerdings wird in Abschnitt $3.6 \mathrm{zu}$ prüfen sein, ob bei den mit einem $w$-Element eingeleiteten Sätzen Merkmal (B) in allen Fällen erfüllt ist, ob also das $w$-Element in der Tat keinen Satzgliedstatus im von ihm eingeleiteten Satz hat. 
Neben der Position in den zur Satzstruktur im engeren Sinne gehörenden Feldern Vorfeld, Nachfeld oder auch Mittelfeld können Verbletztsätze mit semantischem Subjunktor unter bestimmten informationsstrukturellen Bedingungen auch Positionen einnehmen, die außerhalb dieser zentralen Satzstruktur liegen:

(1) a. Wenn der Herbst mit seinen langen Abenden kommt, dann wird es Zeit für alle Leseratten, sich mit Lesestoff einzudecken.

b. Es wird Zeit für alle Leseratten, sich mit Lesestoff einzudecken, liebe Leute, wenn der Herbst mit seinen langen Abenden kommt.

(7) Wenn Sie mich fragen, der Herbst ist bereits da.

In (1a) und (1b) ist der Verbletztsatz aus der zentralen Satzstruktur , herausgestellt' (zu Herausstellung vgl. Altmann 1981). Bei (1a) spricht man dann von ,Linksversetzung in eine Position im ,linken Außenfeld' (vgl. IDS-Grammatik) oder auch im ,Vorvorfeld‘ (Duden-Grammatik 2009: $§ 1384$ ). Charakteristisch ist hier die Wiederaufnahme des Nebensatzes durch ein Adverb wie dann oder so, das als ,Korrelat' fungiert. ${ }^{2}$ Bei (1b) handelt es sich um , Rechtsversetzung ' bzw. einen ,Nachtrag (nach Altmann 1981): Im Nachfeld des Matrixsatzes befindet sich die Infinitivkonstruktion. Auf diese folgt die Anrede liebe Leute, die nicht in den Gesamtsatz integriert ist und dem ,rechten Außenfeld‘ bzw. ,Nachnachfeld‘ zuzuordnen ist. Erst dann folgt der rechtsversetzte wenn-Satz. Um welche Satzposition im weiteren Sinne es sich dann handelt, ist in der Literatur umstritten.

Bei (7) befindet sich der wenn-Satz syntaktisch desintegriert vor dem regulär mit dem Vorfeld beginnenden Hauptsatz. Anders als bei (1a) gibt es kein Korrelat. Syntaktische Desintegration an der linken Peripherie ist charakteristisch für epistemische oder illokutionsbezogene Verwendungen von Nebensätzen (wie in (7)).

\subsection{Morphologische Merkmale}

Zu beachten ist hier lediglich die morphologische Struktur des Subjunktors. Es finden sich sowohl morphologisch einfache Einheiten (wie weil, da, wenn, als) als auch morphologisch komplexe, die erkennbar aus mehreren Morphemen bestehen (wie bevor, indem, nachdem, obgleich, wobei). Neben solchen wortförmigen Subjunktoren (zu einer vollständigen Liste vgl. Fabricius-Hansen 2007: 760) wer-

2 Korrelate in ,Angabesätzen' bilden nach Zitterbart, Satztyp und Korrelat, in diesem Band, Abschnitt 3.2 eine heterogene Gruppe, in der sich die Vielfalt der semantischen Verhältnisse zwischen Matrix- und Untersatz widerspiegeln kann. 
den auch Wortverbindungen zu den Subjunktoren gerechnet wie wenn auch, als ob, auf dass, angenommen, dass (vgl. dazu Fabricius-Hansen 2007: 764ff.).

Generell gilt, dass Subjunktoren in enger Beziehung zu Ausdrücken anderer Wortklassen stehen, ja dass sie im Laufe der Sprachentwicklung durch so genannte ,Grammatikalisierung، aus ihnen hervorgegangen sind: Der Subjunktor $d a$ hat sich aus dem Adverb da entwickelt, der Subjunktor wenn ist eng bezogen auf das Interrogativadverb wann, der Subjunktor als hängt mit der Vergleichspartikel als zusammen. Darin spiegelt sich wider, dass Subjunktoren in einer frühen historischen Stufe des Deutschen noch nicht als eigene Klasse konsolidiert waren. Dies hat auch mit der vergleichsweise späteren Herausbildung subordinierender Syntax überhaupt zu tun, auch in anderen Sprachen lassen sich vergleichbare Entwicklungen nachweisen (vgl. z.B. Kortmann 1998).

In den morphologisch komplexen Subjunktoren ist oft ein Teil enthalten, der sonst als Präposition vorkommt (bevor, indem, nachdem, seitdem, wobei). Transparent sind solche Zusammensetzungen, wenn der andere Bestandteil als Komplement der Präposition verstanden werden kann. Dies ist der Fall z. B. bei indem, nachdem, seitdem, wo auf die Präposition die der Rektion entsprechende Dativform dem folgt, die wir dem Demonstrativpronomen der/die/das zuordnen können. Bei wobei, weswegen geht der erste Bestandteil auf $w$-Elemente zurück, die im Deutschen interrogative oder relative Funktion haben: wo ist Interrogativbzw. Relativadverb, wes die Kurzform des Genitivs des Interrogativ- und Relativpronomens wer/was. Die demonstrativen bzw. relativen Bestandteile der Subjunktoren verweisen hier auf einen der Sachverhalte, die durch den Subjunktor ins Verhältnis gesetzt werden:

nachdem

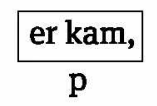

schloss er das Fenster

$\mathbf{q}$

dem verweist auf $\mathrm{p}$ : nach $\mathrm{p}$ ist q der Fall

Er

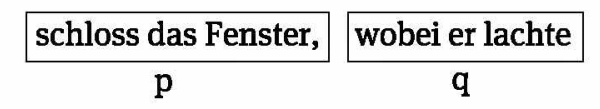

wo verweist auf p: bei p ist q der Fall

\subsection{Kategoriale Merkmale}

Ein kategoriales Merkmal aller Subjunktoren ist, dass sie Verbletztsätze (als Komplement) fordern. Man kann sie (vgl. HDK-1) als Köpfe der von ihnen eingeleiteten Struktur betrachten, die dann Subjunktorphrasen sind. Es handelt sich um exozentrische Phrasen, d.h. der Phrasenkopf ist kategorial verschieden von 
der mit ihm gebildeten Phrase, ähnlich wie bei Präpositionen im Verhältnis zu Präpositionalphrasen. Die Forderungen an das Komplement des Subjunktors beziehen sich, genauer betrachtet, nicht nur auf die Position des Verbs. Das Komplement des Subjunktors ist: ein Satz mit finitem Verb in Letztposition oder anders gesagt: ein finites Verb mit allen Komplementen (einschließlich des Subjekts) und Supplementen/Adjunkten. Andere Merkmale des Verbs, etwa der Verbmodus sind nicht kategorial festgelegt. Konjunktivsetzung ist vielmehr bei einzelnen Subjunktoren semantisch bedingt. So steht bei wenn in kontrafaktischen Bedingungsgefügen sowie bei kontrafaktischen Konsekutivsätzen mit als dass oder kontrafaktischen Vergleichssätzen mit als ob/als wenn in der Regel Konjunktiv II (Präteritum, Plusquamperfekt); vgl. IDS-Grammatik: 1750f. Bei Subjunktoren mit finaler bzw. deontischer Bedeutung wie damit, auf dass kann in gehobenem Register Konjunktiv I (Präsens, Perfekt) stehen; vgl. IDS-Grammatik: 2318.

Komplement eines Subjunktors kann in bestimmten Fällen auch eine nichtsatzförmige Struktur sein, nach HDK-1 (S. 361ff.) ein ,Nichtsatz'. Man kann hier von Ellipsen ausgehen. Dabei entfallen entweder Subjekt und Hilfs- oder Kopulaverb (8) oder das Weggelassene ist aus dem Kontext zu erschließen (9):

(8) Weil von allen geschätzt wird, wurde er zum Sprecher gewählt. Obwohl sie tüchtig und freundlich ist, traut man ihr nicht.

(9) Wenn sie kommt, machen wir einen Spaziergang, wenn sie nicht kømmt, bleiben wir zuhause.

\subsection{Intonatorische Merkmale}

Wir gehen nur knapp vor allem auf den Aspekt der Akzentuierung ein. Zum Aspekt des Tonmusters von Verbletztsätzen mit semantischem Subjunktor wird auf die Darstellungen in der IDS-Grammatik (S. 201) sowie vor allem auf HDK-1 (S. 371ff.) verwiesen. Wesentlich ist hier: Entsprechend ihrem Status als subordinierte Sätze werden solche Nebensätze in der Regel durch ein so genanntes ,progredientes Tonmuster' in den Gesamtsatz integriert.

Allerdings können Subjunktorsätze mit semantischem Subjunktor auch durch finale Grenztonmuster und eine deutliche Pause abgetrennt werden. Dadurch wird ein Status als eigene ,kommunikative Minimaleinheit‘ signalisiert.

Verbletztsätze mit semantischem Subjunktor können zusammen mit ihrem Matrixsatz eine einzige Akzentdomäne bilden. In diesem Fall gilt:

Jeder der Teilsätze hat einen Satzakzent, der bei „normaler Betonung“ (vgl. Höhle 1982) den Regeln der so genannten ,Fokus-Projektion‘ folgt. 
- Einer der beiden Satzakzente ist jedoch stärker hervorgehoben; er ist der Satzakzent des Gesamtsatzes. Die anderen Akzentstellen werden zu ,Nebenakzenten'.

Der Träger des Satzakzents des Gesamtsatzes ist Teil des informationellen Vordergrundes, er ist ,Fokus-Exponent‘. Der Fokus (kurz: ,F') kann dabei unterschiedlich weit ausgedehnt sein (vom Morphem, über die lexikalische Einheit als ,minimalen Foki', die Phrase bis zur ganzen Verbalgruppe bzw. Verbalphase bzw. dem ganzen Teilsatz als ,maximalem Fokus'). Sowohl der Matrixsatz als auch der Nebensatz können den Fokus-Exponenten enthalten, jeder der beiden kann ,fokal' sein. Die übrigen Teile des Gesamtsatzes bilden dann den (noch in sich möglicherweise abgestuften) Hintergrund (kurz: ,H'). In (2a) ist der Nebensatz fokal, in (2b) der Hauptsatz. (Der Träger des Satzakzentes des Gesamtsatzes ist durch Fettdruck + Unterstreichung gekennzeichnet, der Träger eines Nebenakzentes nur durch Unterstreichung.):

(2) a. [A: Warum sind denn die Lager noch gefüllt. B:] [Die Lager sind noch gefüllt]-H [weil der Sommer noch nicht das Gelbe vom Ei war] -F.

b. [Der Sommer war ja bisher nicht so berühmt. B] [Die Lager sind auch noch gefüllt]-F [weil der Sommer noch nicht das Gelbe vom Ei war] -H.

Wenn beide Teilsätze neue oder gleichermaßen relevante Information bringen, können auch beide Teilsätze fokal sein. Nach HDK-1 (S. 372) liegt der „Hauptakzent der Konnektorkonstruktion" hier grundsätzlich im letzten Konnekt:

(2) c. [A: wie ist denn nun die Lage in Ihrem Bereich? B] [weil der Sommer noch nicht das Gelbe vom Ei war] -F [sind die Lager noch gefülltt]-F

Wichtig ist noch folgende Besonderheit. Auch die Subjunktoren selbst können (als minimale Foki) den Hauptakzent tragen:

(10) [A. Ich habe dich doch gewarnt, dieses Unternehmen ist gefährlich. B] [weil -F [es so gefährlich ist]-H [hat es mich gereizt]-F

Bei einer Hervorhebung des formalen Subjunktors dass spricht man von VerumFokus (vgl. Höhle 1992). Dabei wird auf die Tatsache, dass der Sachverhalt besteht, abgehoben. Durch Betonung eines semantischen Subjunktors hingegen wird auf das unerwartete semantische Verhältnis zwischen den Sachverhalten gezielt (vgl. auch Pittner, Adverbialsatz in diesem Band, Abschnitt 2.3.3). 


\subsection{Mögliche syntaktische Funktionen von Verbletztsätzen mit semantischem Subjunktor}

Alle Verbletztsätze mit semantischen Subjunktoren werden als adverbiale Bestimmungen mit Bezug auf den Matrixsatz gebraucht, in der Terminologie der IDS-Grammatik somit als ,Satzadverbialia‘. Dies ist ihre prototypische syntaktische Funktion. Alle bisher genannten Beispiele für Subjunktorsätze waren dieser Art - wobei unter die syntaktische Funktion des Satzadverbials semantisch sowohl propositionsbezogene als auch epistemisch oder illokutionsbezogen zu interpretierende Sätze fallen. Adverbiale Bestimmungen sind in der Regel nicht valenzgebunden; sie können als adverbiale Supplemente (in der Terminologie der IDS-Grammatik) frei zum Matrixsatz hinzugesetzt werden. Bei einem Verb wie dauern ist eine adverbiale Bestimmung der Zeit dagegen durch die Valenz gefordert. Eine Realisierungsform neben anderen (NP, Adjektivphrase, PP) ist der Nebensatz mit dem Subjunktor bis:

(11) Die Sitzung dauert drei Stunden / sehr lange / bis fünf Uhr / bis alle Themen abgearbeitet sind.

In der Terminologie der IDS-Grammatik handelt es sich dann um ein Adverbialkomplement. Auch als Ergänzung zu einem Kopulaverb, somit als Prädikativkomplement, sind gewisse Subjunktorsätze mit einem semantischen Subjunktor möglich, z. B. anstelle eines prädikativen Adjektivs:

(12) Das ist schwierig / als ob man nie fertig würde.

Eine bedeutendere Rolle spielen jedoch vor allem wenn- und wie-Sätze, die als Objekt (Akkusativobjekt (13a, 14) oder Präpositionalobjekt (13b)) bei einem Verb erscheinen, das in der Regel einen dass-Satz für diese Komplementstelle fordert:

(13) a. Ich bedaure sehr, dass ich Sie gekränkt habe / wenn ich Sie gekränkt habe.

b. Wir freuen uns, dass es Ihnen gut geht / wenn es Ihnen gut geht.

(14) Alle sahen, dass er die Treppe herunterging / wie er die Treppe herunterging.

Vor allem bei Nebensätzen mit einem temporalen Subjunktor wie als, wenn, bevor usw. kann auch ein Bezug auf eine nominale bzw. präpositionale Konstituente oder ein Adverb vorliegen. Sie haben dann die syntaktische Funktion des Attributs.

(15) Im Jahr 2003, als das Wetter außergewöhnlich gut war, wurden in den Sommer- und Freibädern 2,4 Millionen Gäste gezählt. (Berliner Zeitung, 12. 05. 2005) 
(16) Immer, wenn es kalt wird, wachsen die Zweifel an der Klimaerwärmung. (St. Galler Tagblatt, 23. 03. 2010)

Handelt es sich bei der Bezugskonstituente um ein semantisch redundantes Adverb wie dann, (polyfunktional) oder deshalb (kausal), so können diese auch als Korrelate zum Subjunktorsatz ohne eigene syntaktische Funktion eingestuft werden, der seinerseits als adverbiale Bestimmung fungiert:

(17) Die Giraffe, glaubte er, hat deshalb einen langen Hals, weil sie ihn Generation um Generation nach immer höheren Blättern streckte. (die tageszeitung, 03. 04. 2009)

\subsection{Verbletztsatz-Einleiter mit syntaktischer Funktion}

Wie in Abschnitt 3.1 angedeutet, gibt es Verbletztsatz-Einleiter, die Kriterium (B), fehlender Satzgliedstatus für das Einleitungselement, nicht klar erfüllen. $w$-Elemente wie wie, wo, wann, warum, wobei, womit, wogegen werden, sofern sie Hauptsätzen einleiten, als Interrogativadverbien mit der syntaktischen Funktion des adverbialen Supplementes oder (seltener) adverbialen Komplementes gebraucht:

(18) Wie/Wo/Wann/Warum/Womit/ hast du das getan? / Wobei hat man dich erwischt?

(19) Wogegen hast du dich eingesetzt?

In vergleichbarer Funktion werden sie auch in subordinierten Sätzen - so genannten ,indirekten Fragesätzen* - gebraucht.

(20) Ich frage dich, wie/wo/wann/warum/womit du das getan hast / wobei man dich erwischt hat / wogegen du dich eingesetzt hast.

Sätze mit $w$-Elementen können aber auch ohne die interrogative (oder allgemeiner propositionsfundierte) Funktion gebraucht werden, man spricht dann von freien Relativsätzen (oder gegenstandsfundierten $w$-Sätzen):

(21) Er spielt, wo/womit schon sein Vater gespielt hat. / Ich kämpfe nur, wogegen zu kämpfen es sich lohnt.

,mit dem Gegenstand, mit dem sein Vater schon gespielt hat / , gegen die Dinge/Verhältnisse, ...

Auch als Attribute zu Substantiven oder Pronomina erscheinen w-Sätze und zwar in Parallele zu beiden Verwendungen auf der Satzebene: 
(22) Die Frage, wie/wo/wann/warum/womit du das getan hast, liegt mir am Herzen.

(23) a. Das Problem, womit wir immer konfrontiert sind, ist (...)

b. Das Problem, mit dem wir immer konfrontiert sind, ist (...)

Attributsätze wie in (23a) bezeichnet man als Relativsätze, womit ist hier Relativadverb. In diesem Fall ist in der Regel eine Ersetzung des $w$-Adverbs durch Präposition + Relativpronomen wie in (23b) möglich.

Ist die Bezugsgröße eines Relativadverbs wie womit, wogegen, wobei ein ganzer Satz, spricht man von ,weiterführenden Relativsätzen':

(24) Die Angelegenheit ist nun abgeschlossen, womit sich alle abfinden müssen / wogegen niemand etwas einzuwenden hatte / wobei jeder zu seinem Recht kam.

In all diesen Fällen hat das $w$-Adverb in dem vom ihm eingeleiteten Verbletztsatz die syntaktische Funktion einer adverbialen Bestimmung. Allerdings werden einige dieser Elemente, allen voran wobei, auch als semantische Subjunktoren bzw. wie im HDK-1 als Postponierer eingeordnet. Die Verwendung von wobei als ,komitativer Subjunktor' (vgl. IDS-Grammatik: 2323), kann als Weiterentwicklung der Funktion als Relativadverb eines weiterführenden Nebensatzes betrachtet werden: Als Subjunktor dient wobei als Verknüpfungselement/Konnektor zwischen Matrix- und Untersatz, während es als Relativadverb den Untersatz adverbial spezifiziert und an den Matrixsatz relativisch anknüpft. Es handelt sich um zwei Perspektiven auf denselben grammatischen Sachverhalt. Für die Subjunktor-Perspektive spricht, dass wobei ein subjunktorales negatives Pendant hat, nämlich ohne dass. Für die Relativadverb-Perspektive spricht, wie bei allen diesen $w$-Ausdrücken die Austauschbarkeit von wo+bei mit $d a+b e i$. Man beachte, dass wohingegen anders als die anderen $w$-Adverbien auf die Funktion in weiterführenden Relativsätzen bzw. als Subjunktor beschränkt ist. Es kommt nicht als Interrogativadverb oder Relativadverb in Attributsätzen bzw. freien Relativsätzen vor.

\section{Theoretische Probleme}

Theoretische Diskussionen um Verbletztsätze mit semantischem Subjunktor richten sich vor allem auf die Besetzung der ,linken Satzperipherie'. Bei der Darstellung in Tabelle 2 haben wir die semantischen Subjunktoren im Feldermodell der Position der linken Satzklammer (LSK) zugeordnet. Die linke Satzklammer ist aber außer im Verbletztsatz durch das finite Verb des Satzes besetzt, nur infinite 
Teile des Verbalkomplexes erscheinen dann in der rechten Satzklammer (RSK). Eine grundlegende Tatsache des Deutschen ist somit die Komplementarität von Verbletztsatz-Einleitern und Finitum als Besetzung für LSK. Vor den ,Komplementierern' dass und $o b$, aber auch vor wenn, weil usw. kann standardsprachlich im Verbletztsatz keine von dessen Konstituenten stehen, ein besetztes Vorfeld ist ausgeschlossen. Im bairischen Dialekt, aber auch im Substandard dieser Region dagegen sind Extraktionen wie in Das wenn ich wüsste! Ins Gefängnis, dass er kommt, glaube ich nicht. durchaus üblich.

Generell ist zum Status topologischer Satzmodelle Folgendes anzumerken. Die Satztopologie kann ganz unterschiedlich gesehen werden: Entweder wird sie in einem deskriptiven Modell als Ergebnis rein empirischer Generalisierungen betrachtet und als eine eigene Strukturebene neben der Strukturebene nach syntaktischen Konstituenten und Funktionen (vgl. z.B. IDS-Grammatik: 959). Oder aber sie ist integraler Bestandteil eines syntaktischen Strukturmodells, bei dem lineare Positionen direkt mit strukturellen Positionen assoziiert sind. Letzteres gilt für generative Modelle. Am Beispiel des nach wie vor einflussreichen ,Prinzipienund-Parameter-Modells` von Chomsky (1991) kann der enge Zusammenhang zwischen dem hierarchischen generativen Satzmodell und linearen Satzpositionen (u.a. für das Deutsche) anschaulich gezeigt werden (vgl. etwa die Darstellung in Wöllstein (2010: 84). Dabei ist für unseren Zusammenhang wichtig, dass LSK (bzw. die Position des Finitums oder des Komplementierers) dem so genannten ,funktionalen Kopf ${ }^{6} \mathrm{C}^{0}$ der CP, somit der obersten ,Satzprojektion' zugeordnet sind. Anders gesagt, in der Position LSK dürfen nach diesem Modell nur , funktionale Elemente oder zumindest ,funktional markierte Elemente erscheinen, also Träger von im engeren Sinne grammatischer Funktion wie Finitheit, Komplementation, keine lexikalischen Köpfe, also Träger rein lexikalisch semantischer Information (wie etwa der Verbstamm von Vollverben). Das bedeutet auch, dass bei Anlehnung an das generative Modell starke Annahmen gelten bezüglich der Besetzung von LSK, während bei einem rein deskriptiven Modell für die Besetzung von LSK im Verbletztsatz allein topologische Regularitäten, vor allem die Verdrängung des Finitums nach RSK und der Abgleich mit den Prinzipien der Mittelfeldbesetzung, ausschlaggebend sind.

Semantische Subjunktoren werden bei einem rein deskriptiven Modell generell LSK zugeordnet: Sie verweisen das Finitum nach RSK und können nicht Teil des Mittelfeldes sein. Auch in generativen Analysen erscheinen sie in der Position $\mathrm{C}^{0}$, somit der strukturellen Entsprechung von LSK. Es ist jedoch zu fragen ob semantische Subjunktoren zu Recht als funktionale Köpfe einzuordnen sind, sind sie doch ähnlich wie Adverbien und Präpositionen (auch) Träger begrifflicher oder lexikalischer Information. Zudem kann auch für sie (wie für die $w / d$-Elemente; vgl. weiter unten) geltend gemacht werden, dass substandardsprachlich 
bestimmte semantische Subjunktoren wie bis ${ }^{3}$, bevor, seit(dem), trotzdem mit formalem dass kombiniert werden können (vgl. Duden-Grammatik 2009: §1693) und andere auch standardsprachlich obligatorisch aus einem ,adverbialen' Bestandteil + dass bestehen (wie als dass, aufdass, anstatt dass, außer dass, ohne dass, so dass).

Verbletztsatz-einleitende $w / d$-Elemente als Besetzung von LSK einzuordnen, ist für generative Analysen noch problematischer; vgl. zu diesem Problem auch Axel Unselbstständiger dass- und ob-VL-Satz, in diesem Band, Abschnitt 1.5. Sie verstoßen gleich gegen zwei Annahmen bzgl. Co: Es handelt sich nicht um Elemente funktionaler Kategorien und es handelt sich nicht um Köpfe, sondern um prinzipiell zu Phrasen erweiterbare Elemente, man denke etwa an die den Relativsatz in (23b) einleitende Präpositionalphrase [mit dem]. Wöllstein (2010: 35) nimmt daher an, in indirekten Fragesätzen und Relativsätzen besetze das $w / d$-Element bzw. die dieses Element enthaltende Phrase das Vorfeld, LSK bleibe unbesetzt, oder generativ gewendet: $\mathrm{C}^{0}$ ist durch ein leeres Element besetzt. Stützend wird auf die substandardsprachliche Besetzung von LSK/CO verwiesen wie etwa in:

(25) Kommt darauf an, mit wem dass sie zu tun haben. (zitiert nach DudenGrammatik 2009: § 1347)

Diese Analyse wird auch als wesentliches Argument zugunsten der so genannten ,Uniformitätshypothese' angeführt, nach der alle Satztypen im Deutschen dieselbe topologische Struktur haben: Verbletztsätze hätten somit durchaus ein Vorfeld, dessen Besetzung nur stärkeren Beschränkungen unterliegt als bei Verbzweitsätzen. Auch für die Elemente, die wir als w-Adverb und/oder semantischen Subjunktor betrachten können (wobei, wohingegen), müsste diese Analyse dann gelten. Alternativ wird aber auch erwogen, $w / d$-Elemente bzw. -Phrasen in Verbletztsätzen als klammeröffnende und gleichzeitig Mittelfeldelemente zu betrachten (Altmann/Hofmann 2008: 72).

Wie bereits erwähnt, sind auch andere semantische Subjunktoren von dem so genannten ,Double-filled-Comp-Effekt' (vgl. Wöllstein 2008: 197) betroffen. Anders als die $w / d$-Elemente sind diese jedoch nicht phrasal. Von einer Zuordnung zum Vorfeld wird daher nicht ausgegangen. Allerdings schlägt Wöllstein (2008) zumindest für die Postponierer eine Analyse als Besetzer einer spezifischen Konnektorenposition $\mathrm{Kon}^{0}$ außerhalb der C-Domäne vor.

Aus rein deskriptiver Sicht hingegen, bei der die Annahme von leeren Ele-

3 bis dass ist über $600 \mathrm{Mal}$ in den Mannheimer Korpora belegt. Dies liegt z. T. an den zahlreichen Zitaten von oder Anlehnungen an die formelhafte Wendung „biss dass der Tode euch scheidet“. 
menten (wie einem leeren $\mathrm{C}^{0}$ ) tunlichst vermieden wird, spricht nichts gegen die Zuordnung zu LSK: Satzpositionen wie LSK werden durch Stellungseinheiten, also potentiell im Satz verschiebbare Satzteile, konstituiert (vgl. IDS-Grammatik: 1499), nicht durch weitergehende Annahmen über Kopf-Status oder Nicht-Phrasalität: LSK ist dann diejenige Position, die das Finitum im Aussagesatz einnimmt oder (exklusiv) diejenigen Stellungseinheiten, die im Verbletztsatz an dessen Stelle erscheinen.

\section{Zusammenfassung}

Verbletztsatz-einleitende Ausdrücke können im Deutschen in drei Gruppen eingeteilt werden: dass/ob (Komplementierer, formale Subjunktoren), $w / d$-Elemente und die hier zur Debatte stehenden Elemente. Für letztere und die von ihnen eingeleiteten Sätze gibt es keine einheitliche Terminologie. Wir schlagen die Termini ,semantischer Subjunktor' und,Verbletztsatz mit semantischem Subjunktor' vor. Semantische Subjunktoren befinden sich in der linken Satzklammer gemäß dem Feldermodell der deutschen Satzstruktur. Die von ihnen eingeleiteten Nebensätze stehen im Vor- oder Nachfeld ihres Matrixsatzes, markierter auch im Mittelfeld. Eine Ausnahme bilden ,postponierende' Subjunktoren wie sodass, wobei, die nur Nachfeldposition zulassen. Semantische Subjunktoren sind häufig morphologisch komplex, typischerweise bestehen sie aus Präposition und deiktischem oder anaphorischem Element (nachdem, wobei). Zentrales kategoriales Merkmal der Subjunktoren ist die syntaktische Forderung nach einem Verbletztsatz - daher wird auch von,Subjunktorphrasen' gesprochen. Verbletztsätze mit semantischem Subjunktor können mit ihrem Matrixsatz eine einzige Akzentdomäne und entsprechend eine Hintergrund-Vordergrund-Einheit bilden, jeder der beiden Sätze kann aber auch für sich ,fokal' sein. Die typische syntaktische Funktion dieser Nebensätze ist die einer nicht valenzgebundenen adverbialen Bestimmung (eines ,adverbialen Supplements'). Aber auch andere Funktionen sind möglich (als Komplement, als Attribut). Einen Grenzfall in mehreren Hinsichten stellen Verbletztsätze mit gewissen w-Elementen dar (wo, wobei, wohingegen). Eine theoretische Herausforderung bilden Verbletztsätze mit semantischem Subjunktor vor allem für generative Ansätze, weil sie den strikten Anforderungen für die ,linke Satzperipherie‘ nicht in jeder Hinsicht genügen. 


\section{Literatur}

Altmann, H. (1981): Formen der „Herausstellung“ im Deutschen: Rechtsversetzung, Linksversetzung, Freies Thema und verwandte Konstruktionen. Tübingen: Niemeyer.

Altmann, H./Hofmann, U. (2008): Topologie fürs Examen. Göttingen: Vandenhoeck \& Ruprecht. Chomsky, N. (1981): Lectures on Government and Binding. Dordrecht: Foris.

Duden-Grammatik (2009): Duden: Die Grammatik. Unentbehrlich für richtiges Deutsch. 8. Aufl. Bd. IV. Hg. von der Dudenredaktion. Mannheim: Dudenverlag.

Eisenberg, P. (2006): Grundriß der deutschen Grammatik. Bd. 1. Der Satz. 3. Aufl. Stuttgart:

Metzler.

Engel, U. (2004): Deutsche Grammatik. Neubearbeitung. München: ludicium.

Eroms, H.-W. (2000): Syntax der deutschen Sprache. Berlin: de Gruyter.

Fabricius-Hansen, C. (2007): Subjunktor. In: Hoffmann, L. (Hg.), Handbuch der deutschen Wortarten. Berlin: de Gruyter, 759-790.

[HDK-1] vgl. Pasch, R. et al. (2003).

Höhle, T. (1982): Explikationen für ,normale Betonung und, normale Wortstellung‘. In: Abraham, W. (Hg.), Satzglieder im Deutschen. Tübingen: Narr, 75-152.

Höhle, T. (1992): Über Verum-Fokus im Deutschen. In: Jacobs, J. (Hg.), Informationsstruktur und Grammatik. Opladen: Westdeutscher Verlag, 112-141.

[IDS-Grammatik] vgl. Zifonun, G. et al. (1997).

Kortmann, B. (1998): Adverbial Subordinators in the Languages of Europe. In: Auwera, J. van der (Hg.), Adverbial Constructions in the Languages of Europe. Berlin: de Gruyter, 457-561.

Pasch, R./Brauße, U./Breindl, E./Wassner, U.H. (2003): Handbuch der deutschen Konnektoren. Berlin: de Gruyter.

Paul, H. (1920): Deutsche Grammatik Bd. IV. Syntax 2. Hälfte. Tübingen: Niemeyer.

Wöllstein, A. (2008): Konzepte der Satzkonnexion. Tübingen: Stauffenburg.

Wöllstein, A. (2010): Topologisches Satzmodell. Heidelberg: Winter.

Zifonun, G./Hoffmann, L./Strecker, B. et al. (1997): Grammatik der deutschen Sprache. 3 Bde.

Berlin: de Gruyter.

Gisela Zifonun 